\title{
CONVERGENCE PROPERTIES FOR THE TIME-DEPENDENT SCHRÖDINGER EQUATION
}

\author{
Peter Sjögren and Per Sjölin
}

\begin{abstract}
Consider the solution to the generalized Schrödinger equation $P u=i \partial u / \partial t$ in the halfspace $\left\{(x, t) \in \mathbf{R}^{n} \times \mathbf{R} ; t>0\right\}$, with initial values $u(x, 0)=f(x)$. Here $P$ is an elliptic operator in the $x$ variables with constant coefficients. Assume that $f$ belongs to the Sobolev space $H_{s}$. When $P=\Delta$, it is known that $s>1 / 2$ implies that $u$ converges to $f$ along almost all vertical lines. We extend this result to an arbitrary $P$ and sharpen it by replacing "almost all" by "quasiall". The values of $u$ must then be made precise in a certain way. A related maximal function estimate is proved.

By means of a counterexample, it is shown that the vertical lines cannot be widened into convergence regions. However, for quasiall boundary points $(x, 0)$, we prove that $u \rightarrow f$ along almost all lines through $(x, 0)$.
\end{abstract}

\section{Introduction and results}

For $f$ belonging to the Schwartz space $\mathcal{S}\left(\mathbf{R}^{n}\right)$ set

$$
u(x, t)=(2 \pi)^{-n} \int_{\mathbf{R}^{n}} e^{i x \cdot \xi} e^{i t|\xi|^{2}} \hat{f}(\xi) d \xi, \quad x \in \mathbf{R}^{n}, t \in \mathbf{R}
$$

where the Fourier transform $\hat{f}$ is defined by

$$
\hat{f}(\xi)=\int_{\mathbf{R}^{n}} e^{-i \xi \cdot x} f(x) d x .
$$

The function $u$ is then a solution to the Schrödinger equation $\Delta u=i \partial u / \partial t$. We set

$$
u^{*}(x)=\sup _{0<t<1}|u(x, t)|, \quad x \in \mathbf{R}^{n}
$$

and also introduce Sobolev spaces $H_{s}=H_{s}\left(\mathbf{R}^{n}\right), s \in \mathbf{R}$, by defining the norm

$$
\|f\|_{H_{s}}=\left(\int_{\mathbf{R}^{n}}\left(1+|\xi|^{2}\right)^{s}|\hat{f}(\xi)|^{2} d \xi\right)^{1 / 2} .
$$

The second author was supported by the Swedish Natural Science Research Council.

AMS classification numbers: 35K $30,42 \mathrm{~B} 25$

doi:10.5186/aasfm.1989.1428 
It is then known that the estimate

$$
\left(\int_{B}\left|u^{*}(x)\right|^{2}\right)^{1 / 2} \leq C_{B}\|f\|_{H_{s}}, \quad f \in \mathcal{S},
$$

holds for all balls $B$ in $\mathbf{R}^{n}$ if $s \geq n / 4$ and if $s>1 / 2$ (see L. Carleson [1], B.E.J. Dahlberg and C.E. Kenig [4], C.E. Kenig and A. Ruiz [5], P. Sjölin [6], and L. Vega [7]). In particular it was proved in [6] that (1.3) holds for $s>1 / 2$, and this result was applied to study the existence almost everywhere of $\lim _{t \rightarrow 0} u(x, t)$ for solutions $u$ to the Schrödinger equation.

We shall here extend these results from [6] in several ways. First we replace $\Delta$ by an elliptic operator $P=-p(D)$, where $D=\left(D_{1}, \ldots, D_{n}\right)$ and $D_{k}=-i \partial / \partial x_{k}$. The polynomial $p$ is real and elliptic, i.e., its pricipal part does not vanish in $\mathbf{R}^{n} \backslash\{0\}$. Its degree $m$ is at least 2. Then if $f \in \mathcal{S}\left(\mathbf{R}^{n}\right)$, the function

$$
u(x, t)=(2 \pi)^{-n} \int e^{i x \cdot \xi} e^{i t p(\xi)} \hat{f}(\xi) d \xi, \quad x \in \mathbf{R}^{n}, t \in \mathbf{R},
$$

solves the Cauchy problem $P u=i \partial u / \partial t, u(\cdot, 0)=f$. With this $u$, we use again (1.2) to define $u^{*}$. We then have the following extension of (1.3).

Theorem 1. If $s>1 / 2$, then

$$
\left\|u^{*}\right\|_{L^{2}(B)} \leq C_{B}\|f\|_{H_{s}}, \quad f \in \mathcal{S}
$$

for any ball $B$ in $\mathbf{R}^{n}$.

This inequality is related to the convergence properties of $u$ at the boundary, when $f \in H_{\boldsymbol{s}}$. Improving the known almost everywhere convergence results, we shall obtain convergence along quasievery vertical line. The capacities to be used are those of Sobolev spaces. They are defined for $s>0$ by

$$
C_{s}(E)=\inf \left\{\|g\|_{2}^{2} ; 0 \leq g \in L^{2}\left(\mathbf{R}^{n}\right), G_{s} * g \geq 1 \text { on } E\right\}, \quad E \subset \mathbf{R}^{n}
$$

Here $G_{s}$ is the Bessel kernel, $\hat{G}_{s}(\xi)=\left(1+|\xi|^{2}\right)^{-s / 2}$. By $C_{s}$-q.e. we mean everywhere except on a set of $C_{s}$-capacity 0 , and similarly for $C_{s}$-q.a. When $s>n / 2$, only the empty set has $C_{s}$-capacity 0 .

A function $f \in H_{s}$ can be written as $f=G_{s} * g$ with $g \in L^{2}$, and conversely. At $C_{s}$-q.a. points $x$, this convolution is well defined in the sense that $G_{s} *|g|(x)<$ $\infty$. One can recover these well-defined values of $f$, knowing $f$ almost everywhere. Indeed, it is easily seen that the means of $f$ in small balls centered at $x$ converge to $G_{s} * g(x)$ if $G_{s} *|g|(x)<\infty$.

We now describe how to make the solution $u$ precise by defining it at sufficiently many points. Let $f \in H_{s}$. For every $t,(1.4)$ defines $u(\cdot, t)$ as an $L^{2}\left(\mathbf{R}^{n}\right)$ 
function, because of Plancherel's theorem. This gives a measurable, a.e. defined function $u$ in $\mathbf{R}^{n} \times \mathbf{R}$. With a point $(x, t)$ as center, we let $B_{x, t}(\delta)$ be the ball in $\mathbf{R}^{n+1}$ of radius $\delta>0$, and

$$
B_{x, t}^{\prime}(\delta)=\left\{\left(x^{\prime}, t\right) ;\left|x^{\prime}-x\right|<\delta\right\}
$$

the horizontal disc. Define the value $u(x, t)$ as the limit as $\delta \rightarrow 0$ of the mean value of $u$ in either $B_{x, t}(\delta)$ or $B_{x, t}^{\prime}(\delta)$, at all points $(x, t)$ where this limit exists. We shall speak of the ball and the disc method. Notice in particular that the disc method for $t=0$ gives us back the $C_{s}$-q.e. defined values of $f$.

Theorem 2. Let $s>1 / 2$ and take $f \in H_{s}$. Define $u$ by means of (1.4), and make $u$ precise by the ball or the disc method. If $0<\varrho<s-1 / 2$, the following holds for $C_{\varrho}$-q.a. $x$ : The function $u$ is defined at every point of the vertical line $\{x\} \times \mathbf{R}$, its restriction to the line is continuous, and its value at $(x, 0)$ is $f(x)$.

We remark that instead of balls $B_{x, t}(\delta)$, it is possible to use half-balls $B_{x, t}(\delta) \cap$ $\left\{\left(x^{\prime}, t^{\prime}\right) ; t^{\prime}>t\right\}$. This is more natural at $t=0$ if one is interested in $u$ for $t>0$ only.

For solutions to initial-value problems in a halfspace $\mathbf{R}^{n} \times \mathbf{R}_{+}$given by kernels like the Poisson or heat kernel, one has convergence in an approach region at almost all boundary points. This means that there exists a strictly increasing function $\gamma: \mathbf{R}_{+} \rightarrow \mathbf{R}_{+}$such that the solution $u(y, t)$ tends to the boundary value at $(x, 0)$ as $(y, t) \rightarrow(x, 0)$ and $|y-x|<\gamma(t)$, for a.a. $x \in \mathbf{R}^{n}$. For our problem, however, there is no such convergence region, except trivially when $f \in H_{s}$ and $s>n / 2$. (In that case, $f$ is continuous and $u$ is a continuous extension of $f$.) The following counterexample is for the standard Schrödinger equation $\Delta u=i \partial u / \partial t$.

Theorem 3. Assume that $\gamma: \mathbf{R}_{+} \rightarrow \mathbf{R}_{+}$is a strictly increasing function. Let $u$ and $f$ be related by (1.1). Then there exists an $f \in H_{n / 2}\left(\mathbf{R}^{n}\right)$ such that $u$ is continuous in $\{(x, t) ; t>0\}$ and

$$
\limsup _{\substack{(y, t) \rightarrow(x, 0) \\|y-x|<\gamma(t), t>0}}|u(y, t)|=+\infty
$$

for all $x \in \mathbf{R}^{n}$.

This means that near the vertical line through every boundary point $(x, 0)$ there can be bad points accumulating at $(x, 0)$, at which $u$ takes values far from $f(x)$. However, the bad points are sparse at most boundary points, in the sense that most lines through $(x, 0)$ do not intersect them. This is the content of our last result.

Theorem 4. For $f \in H_{s}, s>1 / 2$, let $u$ be given by (1.4) and made precise as described above. Let $0<\varrho<s-1 / 2$. Then for $C_{\varrho}$-q.a. $x \in \mathbf{R}^{n}$, the restriction of $u$ to the line $t \rightarrow(x+\alpha t, t)$ is continuous for a.a. $\alpha \in \mathbf{R}^{n}$. 
This of course implies convergence to $f(x)$ along almost all lines through $(x, 0)$, since we know from Theorem 2 that $u(x, 0)=f(x)$.

We prove Theorems 2 and 4 by first showing that $u$ is locally in a mixed Sobolev space. This can also be seen by the method of Constantin and Saut [2], [3].

\section{Proofs for vertical approach}

Proof of Theorem 1. We shall follow the idea in the proof of Theorem 1 in [6]. Choose real functions $\varphi_{0} \in C_{0}^{\infty}\left(\mathbf{R}^{n}\right)$ and $\psi_{0} \in C_{0}^{\infty}(\mathbf{R})$. Instead of $u$ we shall consider

$$
S f(x, t)=\varphi_{0}(x) \psi_{0}(t) u(x, t)
$$

We shall first prove that

$$
\|S f\|_{L^{2}\left(\mathbf{R}^{n+1}\right)} \leq C\|f\|_{H_{-s}}, \quad f \in \mathcal{S},
$$

where $s=(m-1) / 2$. One finds that

$$
\int_{\mathbf{R}^{n}} \int_{\mathbf{R}}|S f(x, t)|^{2} d x d t=\int_{\mathbf{R}^{n}} \int_{\mathbf{R}^{n}} \hat{\varphi}(\eta-\xi) \hat{\psi}(p(\eta)-p(\xi)) \hat{f}(\xi) \overline{\hat{f}(\eta)} d \xi d \eta
$$

where $\varphi=\varphi_{0}^{2}, \psi=\psi_{0}^{2}$. We set

$$
K(\xi, \eta)=(1+|\xi|)^{s}(1+|\eta|)^{s} \hat{\varphi}(\eta-\xi) \hat{\psi}(p(\eta)-p(\xi)) .
$$

Arguing as in [6], we see that to prove (2.2) it suffices to prove that

$$
\int_{\mathbf{R}^{n}}|K(\xi, \eta)| d \eta \leq C, \quad \xi \in \mathbf{R}^{n}
$$

The case $|\xi| \leq 2$ in (2.3) is easy since the $\hat{\varphi}$ factor makes $K$ rapidly decreasing in $\eta$. Now assume that $|\xi|>2$. It is clear that

$$
(1+|\xi|)^{s}(1+|\eta|)^{s} \leq C|\xi|^{2 s}+C|\eta-\xi|^{2 s}
$$

and hence

$$
\begin{aligned}
\int|K(\xi, \eta)| d \eta \leq & C|\xi|^{2 s} \int|\hat{\varphi}(\eta-\xi)||\hat{\psi}(p(\eta)-p(\xi))| d \eta+ \\
& +C \int|\eta-\xi|^{2 s}|\hat{\varphi}(\eta-\xi)||\hat{\psi}(p(\eta)-p(\xi))| d \eta
\end{aligned}
$$


The last integral is bounded because of the $\hat{\varphi}$ factor, and (2.3) follows if we can prove that

$$
\int|\hat{\varphi}(\eta-\xi)||\hat{\psi}(p(\eta)-p(\xi))| d \eta \leq C|\xi|^{-2 s}
$$

We need only deal with large $|\xi|$, and since $\hat{\varphi} \in \mathcal{S}$ it suffices to prove that

$$
\int_{B_{\xi}}|\hat{\varphi}(\eta-\xi)||\hat{\psi}(p(\eta)-p(\xi))| d \eta \leq C|\xi|^{-2 s}
$$

where $B_{\xi}=B\left(\xi ; c_{0}|\xi|\right)=\left\{\eta ;|\eta-\xi|<c_{0}|\xi|\right\}$ and $c_{0}>0$.

To estimate $p(\eta)-p(\xi)$ in $B_{\xi}$, we fix $\xi$ and consider $\operatorname{grad} p$. Let $m$ be the degree of $p$ and $p_{m}$ its principal part. Since $\operatorname{grad} p_{m}$ is homogeneous of degree $m-1$, the ellipticity of $p$ implies that $\operatorname{grad} p_{m} \neq 0$ in $\mathbf{R}^{n} \backslash\{0\}$. With $v=\left|\operatorname{grad} p_{m}(\xi)\right|^{-1} \operatorname{grad} p_{m}(\xi)$, one can therefore choose $c_{0}$ and $c>0$ so that $v \cdot \operatorname{grad} p_{m}>c|\xi|^{m-1}$ in $B_{\xi}$. The constants $c_{0}$ and $c$ do not depend on $\xi$. Since $\operatorname{grad}\left(p-p_{m}\right)$ is of degree at most $m-2$, it follows that

$$
v \cdot \operatorname{grad} p>c|\xi|^{m-1} \text { in } B_{\xi}
$$

for large $\xi$, with a new $c$. We replace $\eta$ by coordinates $\left(s, \eta^{\prime}\right)$ defined by

$$
\eta=\xi+s v+\eta^{\prime}, \quad s \in \mathbf{R}, \quad \eta^{\prime} \perp v .
$$

With $p=p(\eta)=p\left(s, \eta^{\prime}\right)$, this gives $|\partial p / \partial s| \geq c|\xi|^{m-1}$ in $B_{\xi}$. For each $\eta^{\prime}$, there exists an $s_{0} \in \mathbf{R}$ such that

$$
|p(\eta)-p(\xi)| \geq c\left|s-s_{0}\right||\xi|^{m-1} \quad \text { in } B_{\xi},
$$

so that

$$
|\hat{\psi}(p(\eta)-p(\xi))| \leq C\left(1+\left|s-s_{0} \| \xi\right|^{m-1}\right)^{-N}
$$

for any $N$. Also

$$
|\hat{\varphi}(\eta-\xi)| \leq C\left(1+\left|\eta^{\prime}\right|\right)^{-N} .
$$

Integrating in the new coordinates, we obtain (2.5) from these two estimates. Now (2.3) and (2.2) follow.

Setting

$$
\|S f\|_{L^{2}\left(H_{r}\right)}^{2}=\int_{\mathbf{R}^{n}}\|S f(x, \cdot)\|_{H_{r}(\mathbf{R})}^{2} d x
$$

we can write $(2.2)$ as

$$
\|S f\|_{L^{2}\left(H_{0}\right)} \leq C\|f\|_{H_{(1-m) / 2}} .
$$


An estimate for $\partial S f / \partial t$ can be obtained in a similar way, cf. [6]. One finds that

$$
\|S f\|_{L^{2}\left(H_{1}\right)} \leq C\|f\|_{H_{(1+m) / 2}} .
$$

Interpolation yields

$$
\|S f\|_{L^{2}\left(H_{1 / 2+\delta}\right)} \leq C\|f\|_{H_{s}},
$$

where $\delta=\delta(s)>0$ for $s>1 / 2$. But the supremum norm in $\mathbf{R}$ is dominated by the $H_{1 / 2+\delta}(\mathbf{R})$ norm when $\delta>0$. Since $\varphi_{0}$ and $\psi_{0}$ are arbitrary, Theorem 1 follows.

To prepare for the next proof we introduce mixed Sobolev spaces $H_{\varrho, r}$ for $\varrho, r \geq 0$. Define

$$
H_{\varrho, r}=H_{\varrho, r}\left(\mathbf{R}^{n} \times \mathbf{R}\right)=\left(G_{\varrho} \otimes G_{r}\right) * L^{2}\left(\mathbf{R}^{n+1}\right),
$$

where $G_{\varrho}$ and $G_{r}$ are Bessel kernels in $\mathbf{R}^{n}$ and $\mathbf{R}$, respectively. The norm in $H_{\varrho, r}$ is the obvious one. Notice that $H_{0, r}=L^{2}\left(H_{r}\right)$. We start by establishing some properties of $H_{\varrho, r}$, assuming $r>1 / 2$.

Let $*_{1}$ and $*_{2}$ denote convolution in $x$ and in $t$, respectively. If $v \in H_{\varrho, r}$, we can write

$$
v=\left(G_{\varrho} \otimes G_{r}\right) * g=G_{\varrho} *_{1}\left(G_{r} *_{2} g\right)
$$

with $g \in L^{2}\left(\mathbf{R}^{n+1}\right)$. For $r>1 / 2$ one has $G_{r} \in L^{2}(\mathbf{R})$, so that for each $t$

$$
\left|\left(G_{r} *_{2} g\right)(x, t)\right| \leq\left\|G_{r}\right\|_{L^{2}(\mathbf{R})}\|g(x, \cdot)\|_{L^{2}(\mathbf{R})} .
$$

The right-hand side here is in $L^{2}\left(\mathbf{R}^{n}\right)$ as a function of $x$. But then (2.6) says that $x \rightarrow v(x, t)$ is in $H_{\varrho}\left(\mathbf{R}^{n}\right)$ for each $t$. This means that we have a continuous restriction map $R_{t}: H_{\varrho, r} \rightarrow H_{\varrho}\left(\mathbf{R}^{n}\right)$ to each horisontal hyperplane $\mathbf{R}^{n} \times\{t\}$.

Interchanging the variables, we write $v=G_{r} *_{2}\left(G_{\varrho} *_{1} g\right)$. The function $t \rightarrow v(x, t)$ will belong to $H_{r}(\mathbf{R})$ if and only if $t \rightarrow G_{\varrho} *_{1} g(x, t)$ is in $L^{2}(\mathbf{R})$. By Minkowski's inequality,

$$
\left\|G_{\varrho} *_{1} g(x, \cdot)\right\|_{L^{2}(\mathbf{R})} \leq\left(G_{\varrho} *\|g\|_{L^{2}(d t)}\right)(x) .
$$

Here

$$
\|g\|_{L^{2}(d t)}(x)=\left(\int|g(x, t)|^{2} d t\right)^{1 / 2}
$$

is a function in $L^{2}\left(\mathbf{R}^{n}\right)$. But then the right-hand side of $(2.7)$ is in $H_{\varrho}\left(\mathbf{R}^{n}\right)$, hence finite for $C_{\varrho}$-q.a. $x$. We conclude that $t \rightarrow v(x, t)$ is in $H_{r}(\mathbf{R})$, and hence continuous, for $C_{\varrho}$-q.a. $x$. 
We shall say that the value $v(x, t)$ is well defined if

$$
\left(G_{\varrho} \otimes G_{r}\right) *|g|(x, t)<\infty .
$$

What we have just seen implies that this happens for $(x, t) \in E \times \mathbf{R}$, where the complement of $E \subset \mathbf{R}^{n}$ is of $C_{\varrho}$-capacity 0 .

We claim that (2.8) implies

$$
v(x, t)=\lim _{\delta \rightarrow 0} \frac{1}{\left|B_{x, t}(\delta)\right|} \int_{B_{x, t}(\delta)} v\left(x^{\prime}, t^{\prime}\right) d x^{\prime} d t^{\prime}
$$

and similarly for the means in $B_{x, t}^{\prime}(\delta)$. Indeed, set $\chi_{\delta}=|B(\delta)|^{-1} \chi_{B(\delta)}$ with $B(\delta)=B_{0,0}(\delta)$. The mean in $(2.9)$ is then $\chi_{\delta} *\left(G_{\varrho} \otimes G_{r}\right) * g(x, t)$. Clearly, $\chi_{\delta} *\left(G_{\varrho} \otimes G_{r}\right)$ converges pointwise to $\left(G_{\varrho} \otimes G_{r}\right)$ as $\delta \rightarrow 0$. Inscribing $B(\delta)$ in a product of an $n$-dimensional ball and an interval, we obtain a majorization

$$
\chi_{\delta} *\left(G_{\varrho} \otimes G_{r}\right) \leq C G_{\varrho} \otimes G_{r} .
$$

Now (2.8) implies (2.9) via dominated convergence. For $B_{x, t}^{\prime}(\delta)$ we need only use the fact that $x \rightarrow v(x, t)$ is in $H_{\varrho}\left(\mathbf{R}^{n}\right)$.

Proof of Theorem 2. Let $f \in H_{s}$. We write $S f$ for the function obtained when we define $u$ by means of (1.4) and then multiply by $\varphi_{0}(x) \psi_{0}(t)$. Since $\varphi_{0}$ and $\psi_{0}$ are arbitrary, we can replace $u$ by $S f$ in the whole proof.

With $f \in \mathcal{S}$, we first argue as in the preceding proof, using instead of $S f$ its first-order derivatives with respect to $x$. This will produce either an extra $\xi$ factor or a differentiation of $\varphi_{0}(x)$ in the integral expression for $S f$. For $f \in \mathcal{S}$ we get

$$
\left\|\operatorname{grad}_{x} S f\right\|_{L^{2}\left(H_{0}\right)} \leq C\|f\|_{H_{(3-m) / 2}}
$$

and thus

$$
\|S f\|_{H_{1,0}} \leq C\|f\|_{H_{(3-m) / 2}} .
$$

If we differentiate also with respect to $t$, the result will be

$$
\|S f\|_{H_{1,1}} \leq C\|f\|_{H_{(3+m) / 2}} .
$$

This can be combined with our previous estimates in $H_{0,0}=L^{2}\left(H_{0}\right)$ and $H_{0,1}=$ $L^{2}\left(H_{1}\right)$. Interpolating one index at a time, we conclude

$$
\|S f\|_{H_{e, r}} \leq C\|f\|_{H_{e+1 / 2+m(r-1 / 2)}}
$$

for $0 \leq \varrho, r \leq 1$ and $f \in \mathcal{S}$. By means of higher order derivatives, this can actually be extended to arbitrary $\varrho, r \geq 0$. Given $s>1 / 2$ and $0<\varrho<s-1 / 2$, 
we can choose $r>1 / 2$ so that $s=\varrho+1 / 2+m(r-1 / 2)$. Extending $S$, we get a continuous linear map $\bar{S}: H_{s}\left(\mathbf{R}^{n}\right) \rightarrow H_{\varrho, r}\left(\mathbf{R}^{n} \times \mathbf{R}\right)$.

Let $f \in H_{s}$. Then $\bar{S} f$ is a convolution $\left(G_{\varrho} \otimes G_{r}\right) * g, g \in L^{2}$. On $C_{\varrho}$-q.a. vertical lines, this convolution is well defined, with a continuous restriction. It remains to see that its values there coincide with those obtained when we make $S f$ precise. For the ball method, it is enough to verify that $\bar{S} f$ and $S f$ agree a.e. in $\mathbf{R}^{n+1}$, because of the properties of $H_{\varrho, r}$ discussed above. But $\bar{S} f$ and $S f$ define the same function in $L^{2}\left(\mathbf{R}^{n+1}\right)$, since we get two coinciding continuous maps $H_{s} \rightarrow L^{2}\left(\mathbf{R}^{n+1}\right)$. To deal with the disc method, observe that (1.4) gives for any fixed $t$ a continuous map $H_{s}\left(\mathbf{R}^{n}\right) \rightarrow H_{s}\left(\mathbf{R}^{n}\right)$. Multiplying by $\varphi_{0}(x) \psi_{0}(t)$, we conclude that the restriction of $S f$ to $\mathbf{R}^{n} \times\{t\}$ defines a continuous map $H_{s} \rightarrow H_{s}$. This last map agrees with $R_{t} \circ \bar{S}: H_{s} \rightarrow H_{\varrho}$ on $\mathcal{S}$ and thus everywhere. It follows that all the well-defined values of $\bar{S} f$ are obtained when $S f$ is made precise by means of discs.

It only remains to see that the values of $f$, or rather $\psi_{0}(0) \varphi_{0} f$, are recovered $C_{\varrho}$-q.e. in the hyperplane $t=0$ when $S f$ is made precise. Both methods produce the same well-defined values of $\bar{S} f$. But since $\psi_{0}(0) \varphi_{0} f$ is obviously recovered if discs are used, the proof is complete.

\section{Proof for wider approach}

Proof of Theorem 3. We shall first define sequences $\left(R_{j}\right)_{1}^{\infty}$ and $\left(R_{j}^{\prime}\right)_{1}^{\infty}$ such that $2=R_{1}<R_{1}^{\prime}<R_{2}<R_{2}^{\prime}<R_{3}<R_{3}^{\prime}<\cdots$ and points $\left(x_{j}, t_{j}\right) \in \mathbf{R}^{n} \times \mathbf{R}_{+}$. We set $S_{j}=\left\{\xi \in \mathbf{R}^{n} ; R_{j}<|\xi|<R_{j}^{\prime}\right\}$,

$$
\hat{f}(\xi)=|\xi|^{-n}(\log |\xi|)^{-3 / 4} e^{-x_{j} \cdot \xi} e^{-i t_{j}|\xi|^{2}}, \quad \xi \in S_{j},
$$

and $\hat{f}(\xi)=0$ otherwise. It is then clear that $f \in H_{n / 2}$. Our idea is to make $|u|$ large at the points $\left(x_{j}, t_{j}\right)$. Also set $\delta_{k}=\gamma(1 / k) / \sqrt{n}, k=1,2,3, \ldots$ We let $x_{1}, x_{2}, \ldots, x_{n_{1}}$ denote all points $x$ in $B(0 ; 1)=\left\{x \in \mathbf{R}^{n} ;|x|<1\right\}$ such that $x / \delta_{2} \in \mathbf{Z}^{n}, x_{n_{1}+1}, \ldots, x_{n_{2}}$ all points in in $B(0 ; 2)$ such that $x / \delta_{3} \in \mathbf{Z}^{n}$, and generally $x_{n_{k}+1}, \ldots, x_{n_{k+1}}$ all points in $B(0 ; k+1)$ such that $x / \delta_{k+2} \in \mathbf{Z}^{n}$. Then choose $\left(t_{j}\right)_{1}^{\infty}$ such that $1>t_{1}>t_{2}>t_{3}>\cdots>0$ and such that

$$
\frac{1}{k+1}>t_{j}>\frac{1}{k+2}
$$

for $n_{k}+1 \leq j \leq n_{k+1}, k=0,1,2, \ldots\left(n_{0}=0\right)$. Note that the points $\left(x_{j}, t_{j}\right)$ accumulate at each boundary point $(x, 0)$, even if only $\left(x_{j}, t_{j}\right)$ with $\left|x_{j}-x\right|<\gamma\left(t_{j}\right)$ are considered. To define $\left(R_{j}\right)_{1}^{\infty}$ and $\left(R_{j}^{\prime}\right)_{1}^{\infty}$ we first choose $R_{1}=2$ and $R_{1}^{\prime}=3$. Given $R_{1}, R_{1}^{\prime}, \ldots, R_{j-1}, R_{j-1}^{\prime}$ we then choose $R_{j}>R_{j-1}^{\prime}$ such that for $k<j$ one has

$$
R_{j}^{2} \geq C 2^{j} /\left|t_{k}-t_{j}\right|
$$


and

$$
\left|t_{k}-t_{j}\right| R_{j}>\left|x_{k}-x_{j}\right|+1 \text {. }
$$

Also set $R_{j}^{\prime}=R_{j}^{K}$ where $K$ is large.

Now let

$$
u_{m}(x, t)=(2 \pi)^{-n} \int_{|\xi|<\mathbf{R}_{m}^{\prime}} e^{i x \cdot \xi} e^{i t|\xi|^{2}} \hat{f}(\xi) d \xi
$$

Then $u_{m}(\cdot, t) \rightarrow u(\cdot, t)$ in $L^{2}\left(\mathbf{R}^{n}\right)$ for each $t$, and

$$
u_{m}(x, t)=\sum_{j=1}^{m}(2 \pi)^{-n} \int_{S_{j}} e^{i\left(x-x_{j}\right) \cdot \xi} e^{i\left(t-t_{j}\right)|\xi|^{2}}|\xi|^{-n}(\log |\xi|)^{-3 / 4} d \xi=\sum_{j=1}^{m} A_{j}(x, t)
$$

We first observe that

$$
\begin{aligned}
\left|\sum_{j=1}^{k-1} A_{j}(x, t)\right| & \leq \int_{2 \leq|\xi| \leq R_{k-1}^{\prime}}|\xi|^{-n}(\log |\xi|)^{-3 / 4} d \xi \\
& =C \int_{2}^{R_{k-1}^{\prime}} r^{-1}(\log r)^{-3 / 4} d r \leq C\left(\log R_{k-1}^{\prime}\right)^{1 / 4} \leq C\left(\log R_{k}\right)^{1 / 4}
\end{aligned}
$$

for all $(x, t)$. We also have

$$
\begin{aligned}
A_{k}\left(x_{k}, t_{k}\right) & =(2 \pi)^{-n} \int_{S_{k}}|\xi|^{-n}(\log |\xi|)^{-3 / 4} d \xi \\
& =C \int_{R_{k}}^{R_{k}^{\prime}} r^{-1}(\log r)^{-3 / 4} d r=C\left(\left(\log R_{k}^{\prime}\right)^{1 / 4}-\left(\log R_{k}\right)^{1 / 4}\right) \\
& >c\left(\log R_{k}^{\prime}\right)^{1 / 4}, \quad c>0 .
\end{aligned}
$$

For $j>k \geq 2$ one finds that

$$
A_{j}\left(x_{k}, t_{k}\right)=(2 \pi)^{-n} \int_{S^{n-1}} d S\left(\xi^{\prime}\right) \int_{R_{j}}^{R_{j}^{\prime}} r^{-1}(\log r)^{-3 / 4} e^{i F(r)} d r
$$

where

$$
F(r)=\left(x_{k}-x_{j}\right) \cdot \xi^{\prime} r+\left(t_{k}-t_{j}\right) r^{2} .
$$

It follows that

$$
F^{\prime}(r)=\left(x_{k}-x_{j}\right) \cdot \xi^{\prime}+2\left(t_{k}-t_{j}\right) r
$$

and

$$
F^{\prime \prime}(r)=2\left(t_{k}-t_{j}\right) .
$$


Using (3.2) we conclude that

$$
\left|F^{\prime}(r)\right| \geq\left|t_{k}-t_{j}\right| r \geq\left|t_{k}-t_{j}\right| R_{j}, \quad R_{j}<r<R_{j}^{\prime}
$$

and an integration by parts gives

$$
\begin{aligned}
& \int_{R_{j}}^{R_{j}^{\prime}} \frac{1}{r(\log r)^{3 / 4}} e^{i F(r)} d r=\int_{R_{j}}^{R_{j}^{\prime}} \frac{1}{r(\log r)^{3 / 4} i F^{\prime}(r)} i F^{\prime}(r) e^{i F(r)} d r \\
& =\left[\frac{1}{r(\log r)^{3 / 4} i F^{\prime}(r)} e^{i F(r)}\right]_{R_{j}}^{R_{j}^{\prime}}-\int_{R_{j}}^{R_{j}^{\prime}} \frac{d}{d r}\left(\frac{1}{i r(\log r)^{3 / 4} F^{\prime}(r)}\right) e^{i F(r)} d r=A-B .
\end{aligned}
$$

Invoking (3.4) and (3.1), one obtains

$$
|A| \leq \frac{C}{\left|t_{k}-t_{j}\right| R_{j}^{2}} \leq C 2^{-j}
$$

and according to (3.4) and (3.3) we also have

$$
\begin{aligned}
& \left|\frac{d}{d r}\left(\frac{1}{i r(\log r)^{3 / 4} F^{\prime}(r)}\right)\right| \leq C \frac{1}{r^{2}\left|F^{\prime}\right|}+C \frac{\left|F^{\prime \prime}\right|}{r\left|F^{\prime}\right|^{2}} \\
& \leq C \frac{1}{r^{3}\left|t_{k}-t_{j}\right|}+C \frac{\left|t_{k}-t_{j}\right|}{r^{3}\left|t_{k}-t_{j}\right|^{2}}=C \frac{1}{\left|t_{k}-t_{j}\right| r^{3}}
\end{aligned}
$$

and hence

$$
|B| \leq \frac{C}{\left|t_{k}-t_{j}\right| R_{j}^{2}} \leq C 2^{-j}
$$

We conclude that for $j>k$

$$
\left|A_{j}\left(x_{k}, t_{k}\right)\right| \leq C 2^{-j}
$$

It follows that

$$
\left|u_{m}\left(x_{k}, t_{k}\right)\right| \geq c\left(\log R_{k}^{\prime}\right)^{1 / 4}-C\left(\log R_{k}\right)^{1 / 4}-C \sum_{k+1}^{m} 2^{-j} \geq c\left(\log R_{k}^{\prime}\right)^{1 / 4}
$$

when $m>k$ and $K$ is sufficiently large.

To see that $u$ is continuous in $\{t>0\}$, take a compact set $L \subset\{(x, t) ; t>0\}$. Since the sequence $\left(R_{j}\right)$ is very rapidly increasing, there exists a $j_{0}<\infty$ such that (3.1) and (3.2) hold for $j>j_{0}$ with $\left(x_{k}, t_{k}\right)$ replaced by any $(x, t) \in L$. But then one can also take $(x, t) \in L$ instead of $\left(x_{k}, t_{k}\right)$ in $(3.5), j>j_{0}$. Hence, the 
$u_{m}$ converge locally uniformly in $\{t>0\}$. Since each $u_{m}$ is continuous, so is $u$ in $\{t>0\}$. From (3.6) we conclude that

$$
\left|u\left(x_{k}, t_{k}\right)\right| \geq c\left(\log R_{k}^{\prime}\right)^{1 / 4} \rightarrow+\infty
$$

as $k \rightarrow+\infty$. This implies (1.5), and Theorem 3 is proved.

Before the last proof, we must introduce more mixed Sobolev spaces. Fix a large ball $B \subset \mathbf{R}^{n}$. Define a space

$$
H_{\varrho, r, 0}=H_{\varrho, r, 0}\left(\mathbf{R}^{n} \times \mathbf{R} \times B\right)=\left(G_{\varrho} \otimes G_{r}\right) *_{1,2} L^{2}\left(\mathbf{R}^{n} \times \mathbf{R} \times B\right),
$$

with the obvious norm. By $*_{1,2}$ we mean convolution in $\mathbf{R}^{n} \times \mathbf{R}$. The variables will be denoted $x \in \mathbf{R}^{n}, t \in \mathbf{R}, \alpha \in B$.

Let $v=\left(G_{\varrho} \otimes G_{r}\right) *_{1,2} g \in H_{\varrho, r, 0}$ with $r>1 / 2$. For $C_{\varrho}$-q.a. $x$, we claim that for a.a. $\alpha \in B$ the value $v(x, t, \alpha)$ is well defined for all $t \in \mathbf{R}$ and depends continuously on $t$. As before, "well defined" means that the convolution integral is absolutely convergent. We argue as when discussing $H_{\varrho, r}$ in Section 2. Write $v=G_{r} *_{2}\left(G_{\varrho} *_{1} g\right)$. We need only verify that for $C_{\varrho}$-q.a. $x$ the inner convolution here is in $L^{2}(d t)$ for a.a. $\alpha \in B$. But

$$
\left\|G_{\varrho} *_{1} g(x, \cdot, \cdot)\right\|_{L^{2}(\mathbf{R} \times B)} \leq G_{\varrho} *\|g\|_{L^{2}(d t d \alpha)}(x),
$$

and this last quantity is finite for $C_{\varrho}$-q.a. $x$. The claim follows.

Proof of Theorem 4. For $f \in \mathcal{S}$ we write

$$
S^{\prime} f(x, t, \alpha)=S f(x+\alpha t, t)
$$

with $S f$ as before. To deduce an a priori estimate for $S^{\prime} f$, we consider one $\alpha$ at a time and argue as in Section 2. The only difference is that $p(\xi)$ will be replaced by $p(\xi)+\alpha \cdot \xi$. The result is

$$
\left\|S^{\prime} f\right\|_{H_{Q, r}, 0} \leq C\|f\|_{H_{s}}, \quad f \in \mathcal{S} .
$$

Here $\varrho$ and $r$ are as before and $C=C_{B}$. This gives a continuous extension $\bar{S}^{\prime}: H_{s} \rightarrow H_{\varrho, r, 0}$.

We now examine how equality (3.7) extends to $\bar{S}^{\prime} f$. Let $f \in H_{s}$ and take $f_{j} \in \mathcal{S}$ with $f_{j} \rightarrow f$ in $H_{s}$. Then $S^{\prime} f_{j} \rightarrow \bar{S}^{\prime} f$ in $H_{\varrho, r, 0}$. The $H_{\varrho, r, 0}$ norm is given by

$$
\|v\|_{H_{e, r, 0}}^{2}=\int_{B}\|v(\cdot, \cdot, \alpha)\|_{H_{e, r}}^{2} d \alpha
$$

Convergence $v_{j} \rightarrow v$ in $H_{\varrho, r, 0}$ therefore implies that $v_{j}(\cdot, \cdot, \alpha) \rightarrow v(\cdot, \cdot, \alpha)$ in $H_{\varrho, r}$ for a.a. $\alpha$, at least for a subsequence. Restricting to $\mathbf{R}^{n} \times\{t\}$, we get that 
$v_{j}(\cdot, t, \alpha) \rightarrow v(\cdot, t, \alpha)$ in $H_{\varrho}$ for all $t$, for a.a. $\alpha$. On the other hand, $S f_{j}(\cdot+t \alpha, t) \rightarrow$ $S f(\cdot+t \alpha, t)$ in $H_{s}$ because of (1.4). For a.a. $\alpha$, we conclude that for all $t$

$$
\bar{S}^{\prime} f(x, t, \alpha)=S f(x+t \alpha, t), \quad \text { a.a. } \quad x .
$$

When $f \in H_{s}$, we have

$$
\bar{S}^{\prime} f=\left(G_{\varrho} \otimes G_{r}\right) *_{1,2} g \in H_{\varrho, r, 0} .
$$

The property of $H_{\varrho, r, 0}$ deduced before the proof implies that for most $x$ and $\alpha$, the value $\bar{S}^{\prime} f(x, t, \alpha)$ is well defined for all $t$ and depends continuously on $t$. Here "most" is taken in the sense of Theorem 4.

It remains to see that if

$$
\left(G_{\varrho} \otimes G_{r}\right) *_{1,2}|g|(x, t, \alpha)<\infty,
$$

then the value $\left(G_{\varrho} \otimes G_{r}\right) *_{1,2} g(x, t, \alpha)$ is obtained when $S f$ is made precise at the point $(x+t \alpha, t)$. Disregarding those $\alpha$ in a null set, we can assume that (3.8) holds. Notice that $\alpha$ can be kept fixed, since only the restriction $g(\cdot, \cdot, \alpha)$ is used. We know that (3.9) implies that the value of $\left(G_{\varrho} \otimes G_{r}\right) *_{1,2} g$ at $(x, t, \alpha)$ is the limit as $\delta \rightarrow 0$ of the mean of the same function in the disc $B_{x, t}^{\prime}(\delta) \times\{\alpha\}$. But this mean equals the mean of $S f$ in $B_{x+t \alpha, t}^{\prime}(\delta)$, because of (3.8). This settles the case of the disc method.

For the ball method, we see from (3.8) that the mean of $S f$ in $B_{x+t \alpha, t}(\delta)$ equals the mean of $\bar{S}^{\prime} f$ in a set $E_{x, t}^{\alpha}(\delta) \times\{\alpha\}$. Here $E_{x, t}^{\alpha}(\delta)$ is defined by

$$
\left(x^{\prime}, t^{\prime}\right) \in E_{x, t}^{\alpha}(\delta) \quad \Leftrightarrow \quad\left(x^{\prime}+t^{\prime} \alpha, t^{\prime}\right) \in B_{x+t \alpha, t}(\delta) .
$$

But (3.9) implies that the means of $\left(G_{\varrho} \otimes G_{r}\right) *_{1,2} g$ in $E_{x, t}^{\alpha}(\delta) \times\{\alpha\}$ tend to the value of the same function at $(x, t, \alpha)$. This is because $E_{x, t}^{\alpha}(\delta)$ is contained in the ball $B_{x, t}(\sqrt{2}(1+|\alpha|) \delta)$, and its volume is comparable to that of this ball. The dominated convergence argument used for $H_{\varrho, r}$ now carries over. This takes care of the ball method and ends the proof of Theorem 4. 


\section{References}

[1] CARLESon, L.: Some analytical problems related to statistical mechanics. - Euclidean harmonic analysis. Lecture Notes in Mathematics 779. Springer-Verlag, Berlin-Heidelberg-New York, 1979, 5-45.

[2] Constantin, P., and J.-C. Saut: Effets régularisants locaux pour des équations dispersives générales. - C.R. Acad. Sci. Paris Sér. I Math. 304, 1987, 407-410.

[3] Constantin, P., and J.-C. Saut: Local smoothing properties of dispersive equations. Preprint, 1987.

[4] Dahlberg, B.E.J., and C.E. Kenig: A note on the almost everywhere behaviour of solutions to the Schrödinger equation. - Harmonic analysis. Lecture Notes in Mathematics 908. Springer-Verlag, Berlin-Heidelberg-New York, 1982, 205-209.

[5] Kenig, C.E., and A. RuIz: A strong type $(2,2)$ estimate for a maximal operator associated to the Schrödinger equation. -Trans. Amer. Math. Soc. 280, 1983, 239-246.

[6] Sıölın, P.: Regularity of solutions to the Schrödinger equation. - Duke Math. J. 55, 1987, 699-715.

[7] VEGA, L.: Schrödinger equations: pointwise convergence to the initial data. - Proc. Amer. Math. Soc. 102, 1988, 874-878.

Chalmers University of Technology

The University of Göteborg

Uppsala University

Department of Mathematics

Department of Mathematics

S-412 96 Göteborg

Sweden

S-752 38 Uppsala

Sweden

Received 17 September 1987 